ORIGINAL ARTICLE

\title{
Utility of blood cultures in the management of adults with community acquired pneumonia discharged from the emergency department
}

\author{
S G Campbell, T J Marrie, R Anstey, S Ackroyd-Stolarz, G Dickinson
}

See end of article for

Emerg Med J 2003;20:521-523

authors' affiliations

.....................

Correspondence to:

Dr S G Campbell,

Department of Emergency

Medicine, Queen Elizabeth

II Health Sciences Centre,

1796 Summer Street,

Halifax, Nova Scotia,

Canada B3H 3A7;

emsgc@qe2-hsc.ns.ca

Accepted for publication 27 November 2002

Study Objective: To assess the clinical value of blood cultures (BCs) in the management of adult patients discharged from the emergency department (ED) with a diagnosis of community acquired pneumonia (CAP).

Methods: The courses of antibiotic regimens and outcomes of patients with positive $B C$ results were examined to assess their influence on BCs.

Results: BCs were obtained from 289 outpatients. Six clinically significant organisms were identified (a yield of $2.1 \%$ ). Outpatients with CAP who had blood cultures performed had a $0.69 \%$ (2 of 289) chance of having a change of treatment directed by the results of the culture.

....................

Conclusion: BCs have little utility in the ambulatory management of CAP.

D espite the fact that contemporary guidelines for the management of community acquired pneumonia (CAP) recommend ordering blood cultures (BCs) only in admitted patients, ${ }^{1-6}$ BCs are commonly ordered as part of the laboratory investigation of patients with CAP who are subsequently discharged from emergency departments (EDs). The utility of routine $\mathrm{BC}$ has been questioned. ${ }^{7-15} \mathrm{We}$ sought to evaluate the contribution of $\mathrm{BC}$ to patient management and outcome in a large number of Canadian outpatients treated for CAP.

\section{METHODS}

\section{Study design}

The "capitaL study"16 was a multicentre, controlled clinical trial with cluster randomisation to determine if the use of a clinical pathway in the ED improved the efficacy of treatment for CAP without compromising patient wellbeing. We used the data from the capital study to conduct a sub-study to determine whether the use of BC contributed to management or outcome in adult patients with CAP discharged from the ED.

\section{Study setting and population}

Eligible patients were adults discharged from the EDs of participating Canadian hospitals between 1 January and 31 July 1998 after presenting with at least two signs or symptoms of CAP (for example, temperature $>38^{\circ} \mathrm{C}$, productive cough, chest pain, shortness of breath, crackles on auscultation) and whose chest radiograph was compatible with acute pneumonia. Exclusion criteria included pregnant or nursing women, alcohol addiction, chronic renal failure, or immune deficiency. ${ }^{16}$ The study protocol was approved by the research review board of each participating hospital.

\section{Study protocol}

Participating hospitals were assigned either to implement a clinical pathway $(n=9)$, which consisted of a prediction rule to guide the decision regarding the site of treatment, levofloxacin therapy, and practice guidelines, which included BCs being drawn from every patient or continuing conven- tional management of CAP $(n=10)$. In the "intervention" arm, ambulatory therapy was recommended for patients with a Pneumonia Severity Index (PSI), of less than 90, as described by Fine et al. ${ }^{17}$ In the "conventional" arm of the study, the site of treatment and ordering of BCs were left to the discretion of the physician.

BCs were processed at the individual sites. Results were sent to a central data centre, along with other clinical information.

\section{Outcome measures}

The data from patients with positive $\mathrm{BC}$ were analysed by two investigators ( $S C$ and RA) to assess whether the results may have changed the course of treatment for the patient. For the purposes of the study, "utility" of BC was defined as attribution of change in the course of treatment to the $\mathrm{BC}$ result. Any changes in treatment made after 24 hours (before which $\mathrm{BC}$ results are rarely available) were attributed to the BC. "Patient outcome: was defined as hospital admission or death within 42 days of discharge.

\section{RESULTS}

There were 1743 patients enrolled in the capitaL Study. Of these, 1022 were admitted to hospital, and 721 discharged. Blood cultures were drawn from 289 outpatients (40.1\%), 233 of $333(70.0 \%)$ in the intervention arm and 56 of 388 (14.4\%) in the conventional arm, with a yield of six pathogens.

Table 1 shows the courses of treatment of the six patients with positive results. Three patients recovered uneventfully without any change in treatment. One patient, BC positive for Streptococcus pneumoniae with a PSI score of 96, was admitted later the same day of his initial ED discharge. His initial empirical regimen was continued.

Two patients had changes in their course of treatment attributed to $\mathrm{BC}$ results. One patient $\mathrm{BC}$ positive for

Abbreviations: CAP, community acquired pneumonia; $B C$, blood culture; ED, emergency department 
Table 1 Course of patients with positive $B C(n=6)$

\begin{tabular}{|c|c|c|c|c|c|c|}
\hline Organism & Age & $\begin{array}{l}\text { PSI } \\
\text { score }\end{array}$ & First antibiotic & Second antibiotic & $\begin{array}{l}\text { Change in } \\
\text { relation to } \\
\mathrm{BC}\end{array}$ & $\begin{array}{l}\text { Subsequent } \\
\text { admission }\end{array}$ \\
\hline S pneumoniae & 54 & 89 & Levofloxacin & - & None & - \\
\hline$S$ pneumoniae & 44 & 44 & Cefuroxime+Levofloxacin & - & None & - \\
\hline$S$ pneumoniae & 30 & 40 & $\mathrm{TMP} / \mathrm{SMX}$ & $\begin{array}{l}\text { Ceftriaxone/ } \\
\text { erythromycin }\end{array}$ & Possible & 1 day later \\
\hline S pneumoniae* & 66 & 96 & Azithromycin+Cefuroxime & - & None & Same day \\
\hline E coli & 76 & 66 & Levofloxacin & Gentamicin $\rightarrow$ TMP/SMX & Yes & 2 days later \\
\hline E coli & 80 & 90 & Levofloxacin & - & None & - \\
\hline
\end{tabular}

PSI, Pneumonia Severity Index; TMP/SMX, trimethoprim-sulfamethoxazole. *The one positive result found in the conventional arm.

$S$ pneumoniae, initially discharged from the ED receiving treatment with trimethoprim-sulfamethoxazole, was admitted the following day and treated with ceftriaxone and erythromycin. The other patient, BC positive for Escherichia coli, was admitted two days later and treatment was changed to gentamicin followed by oral trimethoprim-sulfamethoxazole on discharge from hospital, one day after admission.

At 42 day follow up, all patients with positive BC were alive and had had no subsequent readmissions. There were no deaths or other readmissions in any other outpatients.

Patients discharged from the ED with CAP who had BC performed had a $0.69 \%$ ( 2 of 289 ) chance of having a change of treatment directed by the results of the BC. Patients in whom BC did yield positive results had only a $33.3 \%$ (2 of 6 ) chance of having a change in treatment determined by the result.

\section{DISCUSSION}

The issue of blood cultures in outpatient CAP management has been neglected in the literature. Stuurman and colleagues, in a retrospective study of all patients (not only CAP) discharged from an ED after BC, found similar results to ours; in $1.8 \%$ of cases (24 of 1350), positive results were found, and $0.52 \%$ ( 7 of 1350 ) of patients had results that potentially affected management. ${ }^{18}$

The advantage of using data from the capitaL Study was that, by the protocol directing $\mathrm{BC}$ in all patients in the intervention arm, we were able to follow up BCs in a large number of outpatients, while the conventional arm would show how many BCs are drawn on this population in current practice.

In the conventional arm, $14.4 \%$ of patients had BCs drawn, demonstrating that BCs are used sporadically, although not insignificantly, in outpatient CAP management. The combined $40 \%$ rate of $\mathrm{BC}$ probably represents selection bias in that sicker patients are more likely to have BCs drawn. Considering that outcomes in all outpatients were favourable, we can assume it to be unlikely that BC would have significantly served any of the patients in whom BCs were not drawn.

In our study, one patient, BC positive for $E$ coli and initially treated with levofloxacin, was subsequently admitted two days later and treated with gentamicin, followed by oral trimethoprim-sulfamethoxazole on discharge from hospital the next day. The investigators considered it probable that the $\mathrm{BC}$ result steered the change in treatment to that with a narrower spectrum of coverage. Although the decision to admit the patient may have been made on clinical grounds, the fact that the patient was discharged one day after his admission suggests that this decision was made as a result of the identification of $E$ coli bacteraemia. The investigators felt that this reason for admission, after two days of treatment on a drug with high oral bioavailability and good $E$ coli coverage, ${ }^{19}$ would have been inappropriate and argue that not receiving the result would have saved the hospital money and the patient inconvenience.

In the case of the patient BC positive for $S$ pneumoniae, although the investigators attributed the admission the following day to the $\mathrm{BC}$ result, the antimicrobial regimen was changed to one with a broader spectrum of coverage than the identification of $S$ pneumoniae would have suggested (ceftriaxone and erythromycin), suggesting that the "step up" in treatment was independent of the BC result.

\section{Limitations}

Our study does have several limitations. Firstly, it is possible that patients excluded from the study may have been candidates for outpatient therapy, so our findings may not apply to all outpatients with CAP.

The fact that $70 \%$ of patients in the intervention arm had BCs suggests poor compliance with the study protocol but, considering the universally favourable outcomes, probably represents selection bias in favour of our conclusions in that these are likely to represent the sickest patients.

Our assessments of the effect of BC on clinical decision making were made using the standard submission form and not the original patient record. To be certain that the influence of BC was not underestimated, any change in antimicrobial class after 24 hours was deemed to have been as a result of the $\mathrm{BC}$, even in cases where the investigators believed that the change was an inappropriate reaction to the $\mathrm{BC}$ result.

\section{Conclusion}

This study evaluated the utility of BCs in the outpatient management of CAP from 19 different Canadian hospitals. Blood cultures rarely contribute to the outpatient management of CAP and should not be used.

\section{Authors' affiliations}

S G Campbell, S Ackroyd-Stolarz, Department of Emergency Medicine, Dalhousie University, Halifax, Nova Scotia, Canada

T J Marrie, Department of Medicine, University of Alberta, Edmonton, Alberta, Canada

R Anstey, Endpoint Research Ltd, Mississauga, Ontario, Canada G Dickinson, Department of Emergency Medicine, University of Ottawa, Ottawa, Ontario, Canada

Presented at the 9th International Conference of Emergency Medicine, Edinburgh, Scotland, 17-21 June 2002.

Funding: this research was supported by grant 9807PT-39621-UI-D from the Medical Research. Council of Canada and by the Pharmaceutical Manufacturers' Association of Canada Awards Program/Industry Partnership (Janssen Ortho Inc was the industry partner).

Conflicts of interest: none declared. 


\section{REFERENCES}

1 Bartlett JG, Breiman RF, Mandell LA, et al. Community-acquired pneumonia in adults: guidelines for management. Clin Infect Dis 1998;26:81 1-38.

2 Feldman C, Bateman ED, Klugman KP, et al. Management of communityacquired pneumonia in adults. South Afr Med J 1996;86:1152-63.

3 Finch RG, Woodhead MA. Practical considerations and guidelines for the management of community-acquired pneumonia. Drugs 1998;55:31-45.

4 San Pedro GS, Campbell GD. Limitations of diagnostic testing in the initial management of patients with community-acquired pneumonia. Semin Resp Infect 1997; 12:300-7.

5 Niederman MS, Bass JB, Campbell GD, et al. Guidelines for the initial management of adults with community-acquired pneumonia: diagnosis, assessment of severity, and initial antimicrobial therapy. Am Rev Respir Dis 1993; 148:1418-26.

6 Mandell LA, Marrie TJ, Grossman RF, et al. Canadian guidelines for the initial management of community-acquired pneumonia: an evidence-based update by the Canadian Infectious Diseases Society and the Canadian Thoracic Society. Clin Infectious Dis 2000;31:383-421.

7 Levy M, Dromer F, Brion N, et al. Community-acquired pneumonia: importance of initial noninvasive bacteriologic and radiographic investigations. Chest 1988;92:43-8.

8 Chalasani NP, Valdecanas MAL, Gopal AK, et al. Clinical utility of blood cultures in adult patients with community-acquired pneumonia without defined underlying risks. Chest 1995;108:932-8.

9 Hickey RW, Bowman MJ, Smith GA. Utility of blood cultures in pediatric patients found to have pneumonia in the emergency department. Ann Emerg Med 1996;27:721-5.
10 Bates DW, Goldman L, Lee TH. Contaminant blood cultures and resource utilization. The true consequences of false-positive results. JAMA 1991;25:365-9.

11 Woodhead MA, Arrowsmith J, Chamberlain-Webber R, et al. The value of routine microbiological investigation in community-acquired pneumonia. Respir Med 1991;85:313-17.

12 Ewig S, Baver T, Hasper E, et al. Value of routine microbial investigation in community-acquired pneumonia treated in a tertiary care centre. Respiration 1996:63:164-9.

13 Waterer GW, Wunderlink RG. The influence of the severity of communityacquired pneumonia on the usefulness of blood cultures. Respir Med 2001;95:78-82.

14 Theerthakarai R, El-Halees W, Ismail $M$, et al. Non-value of the initial microbiological studies in the management of non-severe community-acquired pneumonia. Chest 2001;119:181-4.

15 Sanyal S, Smith PR, Saha AC, et al. Initial microbiologic studies did not affect outcome in adults hospitalized with community-acquired pneumonia Am J Respir Crit Care Med 1999;160:346-8.

16 Marrie TJ, Lau CY, Wheeler SL, et al. A controlled trial of a critical pathway for treatment of community-acquired pneumonia. JAMA 2000;283:749-55.

17 Fine MJ, Auble TE, Yealy DM, et al. A prediction rule to identify low-risk patients with community-acquired pneumonia. N Engl J Med 1997;336:243-50.

18 Stuurman KM, Bopp J, Molinari D, et al. Blood cultures in adult patients released from an urban emergency department: a 15-month experience. Acad Emerg Med 1996;3:768-75.

19 Zhanel GG, Walkty A, Vercaigne L, et al. The new fluoroquinolones: a critical review. Can J Infect Dis 1999;19:207-38. 